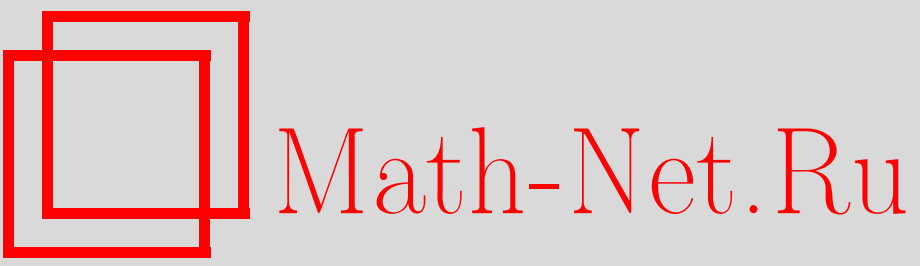

А. В. Маршаков, Матричная модель и стационарная задача в цепочке Тоды, TMФ, 2006, том 146, номер 1, 3-16

DOI: https://doi.org/10.4213/tmf2004

Использование Общероссийского математического портала Math-Net.Ru подразумевает, что вы прочитали и согласны с пользовательским соглашением

http://www . mathnet.ru/rus/agreement

Параметры загрузки:

IP: 54.197 .130 .99

26 апреля 2023 г., 15:53:05 
ТЕОРЕТИЧЕСКАЯ

И МАТЕМАТИЧЕСКАЯ

ФИЗИКА

Том 146, № 1

январь, 2006

(C) 2006 г.

А. В. Маршаков*

\section{МАТРИЧНАЯ МОДЕЛЬ И СТАЦИОНАРНАЯ ЗАДАЧА В ЦЕПОЧКЕ ТОДЫ}

Рассмотрена стационарная задача в цепочке Тоды и продемонстрировано, что возникающие в ней геометрические структуры в точности отвечают многосвязному решению одноматричной модели с полиномиальным потенциалом. Для простейших нетривиальных примеров гамильтонианы и симплектические формы вычислены явно, и полученные формулы проверены на самосогласованность. Сформулирована соответствующая квантовая задача и обсуждены некоторые ее свойства и перспективы.

Ключевые слова: матричные модели, комплексная геометрия, иинтегрируемые системы.

\section{1. ВВЕДЕНИЕ}

Уже достаточно давно было обнаружено (см., например, работы [1], [2]), а впоследствии особо отмечено в контексте струнной геометрии и вытекающих из нее следствий для суперсимметричных калибровочных теорий [3], [4] , что решение одноматричной модели

$$
Z=\int_{N \times N} d \Phi \exp \left(-\frac{1}{\hbar} \operatorname{Tr} W(\Phi)\right)
$$

с полиномиальным потенциалом

$$
W(\Phi)=\sum_{k=1}^{n+1} t_{k} \Phi^{k}
$$

в планарном приближении $(1 / N)$-разложения формулируется геометрически в терминах гиперэллиптической комплексной кривой

$$
y^{2}=W^{\prime}(\lambda)^{2}-4 f_{n-1}(\lambda)
$$

оснащенной производящим дифференциалом (связанным с плотностью распределения собственных значений матрицы $\Phi)$

$$
d S=y d \lambda
$$

* Физический институт им. П. Н. Лебедева, Институт теоретической и эксперименталльной физики, Москва, Россия. E-mail: mars@lpi.ru, mars@itep.ru 
Параметры $t_{k}$ потенциала (2) в данном подходе фиксированы и являются казимирами, в то время как динамические переменные связаны с нетривиальными периодами производящего дифференциала (4) на гиперэллиптической кривой (3).

Такая геометрическая картина предполагает, в частности, что наиболее эффективным способом описания планарного решения матричной модели (1) является теория интегрируемых систем. Действительно, уже в работе [2] было отмечено, что статсумма матричной модели (1) $\mathcal{F}=\hbar^{2} \ln Z$ в пределе $N \rightarrow \infty$ при фиксированном $\hbar N$ формулируется геометрически в терминах (логарифма) квазиклассической тау-функции [5]. Данная конструкция представляет собой частный случай так называемых алгебро-геометрических голоморфных интегрируемых систем, часто возникающих в последнее время в контесте теории струн или теории Виттена-Зайберга (см. [6], [7] и списки литературы в этих книгах).

С другой стороны, хорошо известно, что матричный интеграл (1) как функция параметров потенциала (2) и размера матрицы $N$ есть тау-функция (полубесконечной “ограниченной”) цепочки Тоды [8]. Цепочки Тоды представляют собой хорошо известное семейство интегрируемых моделей, являющихся частным случаем алгебро-геометрических интегрируемых систем: аффинные или периодические версии цепочек Тоды буквально отвечают интегрируемым системам Виттена-Зайберга, ассоциированным с "чистыми" ( $\mathcal{N}=2$ суперсимметричными) калибровочными теориями [9]. При этом возникает естественный вопрос: связана ли интегрируемая система, определяемая геометрией $(3),(4)$, с семейством моделей Тоды?

В данной работе будет продемонстрировано, что комплексная кривая (3), оснащенная дифференциалом (4), непосредственно отвечает конкретной интегрируемой задаче в цепочке Тоды, а именно, стационарной задаче в цепочке Тоды ${ }^{1)}$. Мы обсудим общую интегрируемую структуру классической стационарной задачи и приведем явные вычисления для простейших нетривиальных примеров порядка $n=2,3$, а также кратко обсудим проблемы ее квантования. Мы надеемся, что обнаруженная эквивалентность геометрий матричной модели и стационарной задачи в цепочке Тоды окажется первым шагом к выявлению правильных аналогов дополнительных квазиклассических переменных, а рассмотренная ниже модель будет играть роль удачного модельного примера.

\section{2. ОБОЗНАЧЕНИЯ}

Оператор Лакса цепочки Тоды представляет собой бесконечную трехдиагональную матрицу с элементами

$$
L_{i j}=r_{i} \delta_{j, i-1}+p_{i} \delta_{j i}+r_{i+1} \delta_{j, i+1}, \quad i, j \in \mathbb{Z} .
$$

Введем обозначения

$$
\begin{aligned}
r_{i} & =e^{\left(q_{i}-q_{i-1}\right) / 2}, \\
R_{i} & =r_{i}^{2}=e^{q_{i}-q_{i-1}},
\end{aligned}
$$

\footnotetext{
1) Аналогичная стационарная задача для иерархии КдФ хорошо известна и описана в литературе [10].
} 
удобные при дальнейшем рассмотрении.

Уравнения движения цепочки Тоды следуют из представления Лакса

$$
\frac{\partial L}{\partial t_{1}}=\left[L, A_{1}\right]
$$

где $A_{1}=\mathcal{R} \circ L / 2$, с учетом явного представления для $\mathcal{R}$-матрицы

$$
\left(A_{1}\right)_{i j}=\frac{1}{2}\left(r_{i+1} \delta_{j, i+1}-r_{i} \delta_{j, i-1}\right) .
$$

При этом (8) эквивалентно хорошо известным уравнениям движения

$$
\begin{aligned}
& \frac{\partial r_{i}}{\partial t_{1}}=r_{i}\left(p_{i}-p_{i-1}\right), \\
& \frac{\partial p_{i}}{\partial t_{1}}=r_{i+1}^{2}-r_{i}^{2}=R_{i+1}-R_{i}
\end{aligned}
$$

для цепочки частиц с экспоненциальным взаимодействием между ближайшими соседями.

Стандартным образом нелинейные уравнения (10) могут быть представлены как условие совместности вспомогательной линейной задачи

$$
(L \psi)_{i}=r_{i+1} \psi_{i+1}+p_{i} \psi_{i}+r_{i} \psi_{i-1}=\lambda \psi_{i} .
$$

Базис двумерного пространства решений вспомогательной линейной задачи может быть описан разложениями

$$
\psi_{i}^{ \pm}=\lambda^{ \pm i} e^{\mp \frac{1}{2} q_{i}}\left(1+\frac{\xi_{i}^{ \pm}}{\lambda}+\frac{\eta_{i}^{ \pm}}{\lambda^{2}}+\cdots\right),
$$

где для коэффициентов разложения имеют место формулы

$$
\xi_{i-1}^{+}-\xi_{i}^{+}=p_{i-1}, \quad \xi_{i}^{-}-\xi_{i-1}^{-}=p_{i},
$$

а также

$$
\begin{aligned}
& \eta_{i-1}^{+}-\eta_{i}^{+}=R_{i-1}+\xi_{i-1}^{+}\left(\xi_{i-1}^{+}-\xi_{i}^{+}\right), \\
& \eta_{i}^{-}-\eta_{i-1}^{-}=R_{i+1}+\xi_{i}^{-}\left(\xi_{i}^{-}-\xi_{i-1}^{-}\right),
\end{aligned}
$$

и это непосредственно связывает их с фазовыми переменными цепочки Тоды.

\section{3. СТАЦИОНАРНАЯ ЗАДАЧА}

Бесконечная цепочка Тоды является вполне интегрируемой системой с бесконечным количеством коммутирующих с представлением (8) интегралов движения, которые генерируют высшие потоки. Высшие потоки можно определить следующим образом:

$$
\frac{\partial L}{\partial t_{k}}=\left[L, A_{k}\right],
$$

где $A_{k}=\mathcal{R} \circ L^{k} / 2$ с той же $\mathcal{R}$-матрицей, что и в формуле $(9)$. Существует много способов ограничить бесконечную цепочку Тоды на конечномерное подпространство 
ее фазового пространства; наиболее известными являются $N$-периодическая цепочка и открытая цепочка (“тодовская молекула") из $N$ частиц.

Ниже мы собираемся сконцентрировать свое внимание на стационарной задаче, определяемой условием коммутативности

$$
[L, A]=0,
$$

где

$$
A=\sum_{k=1}^{n} c_{k} A_{k}
$$

при некоторых фиксированных "порядке" $n$ и коэффициентах $\left\{c_{k}\right\}$.

Как в любой задаче коммутативности двух операторов, в стационарной задаче (16) возникает комплексная кривая

$$
\operatorname{det}(A(\lambda)-Y)=0
$$

где матрица

$$
A(\lambda)=\left(\begin{array}{ll}
a(\lambda) & b(\lambda) \\
c(\lambda) & d(\lambda)
\end{array}\right)
$$

с некоторыми полиномиальными матричными элементами является записью оператора (17) в базисе двумерного пространства собственных функций задачи (11) для оператора (5). Поэтому кривая (18) является гиперэллиптической,

$$
y^{2}=P_{2 n}(\lambda) \equiv(a-d)^{2}+4 b c
$$

где $y=2 Y-\operatorname{Tr} A(\lambda)=2 Y-a-d$.

Наиболее естественной симплектической формой для бесконечной цепочки Тоды является $\Omega_{\infty}=\sum_{i \in \mathbb{Z}} \delta q_{i} \wedge \delta p_{i}$. Хорошо известно, что эта формула остается неизменной при редукции на $2 N$-мерное фазовое пространство $N$-периодической задачи или $N$-частичной открытой “молекулы”, т.е. $\Omega_{N}=\sum_{i=1}^{N} \delta q_{i} \wedge \delta p_{i}$. Последнюю формулу можно переписать также в виде

$$
\Omega_{N}=\underset{w=\infty}{\operatorname{res}}\left(\frac{d w}{w} \operatorname{Tr}\left(\Psi^{-1} \delta L(w) \wedge \delta \Psi\right)\right)
$$

в терминах зависящего от спектрального параметра оператора Лакса $N$-периодической задачи, см. подробности в работах [6], [11].

Симплектическая структура стационарной задачи может быть определена аналогичной формулой

$$
\Omega=\underset{\lambda=\infty}{\operatorname{res}}\left(d \lambda \operatorname{Tr}\left(\Psi^{-1} \delta A(\lambda) \wedge \delta \Psi\right)\right),
$$

где теперь $\delta A(\lambda)$ обозначает вариацию (при фиксированном спектральном параметре $\lambda$ !) оператора, определяемого формулами (17), (19), а

$$
\Psi=\left(\begin{array}{cc}
\psi_{i}^{+} & \psi_{i}^{-} \\
\psi_{i-1}^{+} & \psi_{i-1}^{-}
\end{array}\right)
$$


обозначает матричную функцию Бейкера-Ахиезера, для которой $\Psi^{-1}$ является просто обратной матрицей.

В дальнейшем мы используем более удобную и отличную от (12) нормировку функций Бейкера-Ахиезера:

$$
\Psi \rightarrow \Psi\left(\begin{array}{cc}
e^{\frac{q_{i}}{2}} & 0 \\
0 & e^{-\frac{q_{i-1}}{2}}
\end{array}\right) .
$$

Отметим, что нормировка функций Бейкера-Ахиезера не влияет на симплектическую форму (21), так как разность двух выражений, скажем, при изменении нормировки (23), пропорциональна

$$
\operatorname{res}(\delta Y d \lambda) \wedge \delta\left(q_{i}-q_{i-1}\right)=0 .
$$

\section{4. ЯВНЫЕ ВЫЧИСЛЕНИЯ}

Проиллюстрируем теперь общую конструкцию конкретными явными примерами.

4.1. Случай $n=1$. Это тривиальный случай стационарности оператора (9), который в базисе собственных функций (11) принимает вид

$$
A_{1}(\lambda)\left(\begin{array}{c}
\psi_{i} \\
\psi_{i-1}
\end{array}\right)=\left(\begin{array}{cc}
\frac{1}{2}\left(\lambda-p_{i}\right) & -r_{i} \\
r_{i} & -\frac{1}{2}\left(\lambda-p_{i-1}\right)
\end{array}\right)\left(\begin{array}{c}
\psi_{i} \\
\psi_{i-1}
\end{array}\right)
$$

что приводит к кривой нулевого рода (см. формулу (20))

$$
y^{2}=\lambda^{2}-\left(p_{i}+p_{i-1}\right) \lambda+\frac{1}{4}\left(p_{i}+p_{i-1}\right)^{2}+4 R_{i}
$$

и разложению

$$
y d \lambda \underset{\lambda \rightarrow \infty}{\sim} d \lambda\left(\lambda-\frac{1}{2}\left(p_{i}+p_{i-1}\right)+\frac{2 R_{i}}{\lambda}+\cdots\right) .
$$

Условие коммутативности $\left[L, A_{1}\right]=0$ приводит к дальнейшим ограничениям:

$$
p_{i+1}=p_{i}=p_{i-1}, \quad R_{i+1}=R_{i},
$$

что полностью фиксирует коэффициенты уравнений (26) или (27), при этом все переменные превращаются в константы.

В тривиальном случае $n=1$ явное вычисление симплектической формы (21) приводит к формуле

$$
\Omega_{1}=e^{q_{i}-q_{i-1}} d q_{i} \wedge d q_{i-1}+\frac{1}{2}\left(d \xi_{i}^{+} \wedge d \xi_{i}^{-}+d \xi_{i-1}^{+} \wedge d \xi_{i-1}^{-}\right)-d \xi_{i}^{+} \wedge d \xi_{i-1}^{-} .
$$

Поскольку в силу (13) имеет место равенство

$$
\frac{1}{2}\left(d \xi_{i}^{+} \wedge d \xi_{i}^{-}+d \xi_{i-1}^{+} \wedge d \xi_{i-1}^{-}\right)-d \xi_{i}^{+} \wedge d \xi_{i-1}^{-}=\frac{1}{2}\left(d \xi_{i}^{+} \wedge d p_{i}+d p_{i-1} \wedge d \xi_{i-1}^{-}\right),
$$

учитывая условия (28), заключаем, что симплектическая форма (29) обращается в нуль. 
4.2. Случай $n=2$. Обратимся теперь к простейшему нетривиальному случаю $c_{k} \propto \delta_{k n}$ для $n=2$, т.е. к случаю $A=A_{2}=\mathcal{R} \circ L^{2} / 2$. Применяя дважды оператор (5), получим

$$
\begin{gathered}
\left(L^{2} \psi\right)_{i}=r_{i+2} r_{i+1} \psi_{i+2}+r_{i+1}\left(p_{i+1}+p_{i}\right) \psi_{i+1}+\left(R_{i+1}+p_{i}^{2}+R_{i}\right) \psi_{i}+ \\
+r_{i}\left(p_{i}+p_{i-1}\right) \psi_{i-1}+r_{i} r_{i-1} \psi_{i-2}
\end{gathered}
$$

или

$$
\left(A_{2} \psi\right)_{i} \propto r_{i+2} r_{i+1} \psi_{i+2}+r_{i+1}\left(p_{i+1}+p_{i}\right) \psi_{i+1}-r_{i}\left(p_{i}+p_{i-1}\right) \psi_{i-1}-r_{i} r_{i-1} \psi_{i-2}
$$

в то время как условие стационарности градиента $\operatorname{Tr} L^{2}=\sum_{i}\left(p_{i}^{2}+2 R_{i}\right)$ в (31), не дающего вклада в оператор (32), приводит в точности к условиям связи (28) предыдущего тривиального примера.

Условия коммутативности в данном примере приводят к равенствам

$$
R_{i+1}\left(p_{i+1}+p_{i}\right)=R_{i}\left(p_{i}+p_{i-1}\right), \quad R_{i+1}+p_{i}^{2}=R_{i-1}+p_{i-1}^{2},
$$

которые редуцируют размерность фазового пространства до четырех независимых переменных ( $R_{i}$ и $p_{i}$ на двух соседних узлах).

Теперь, используя (11), легко переписать оператор (32) в базисе собственных функций оператора Лакса (5):

$$
A_{2}(\lambda)\left(\begin{array}{c}
\psi_{i} \\
\psi_{i-1}
\end{array}\right)=\left(\begin{array}{cc}
\frac{1}{2}\left(\lambda^{2}-p_{i}^{2}-R_{i+1}+R_{i}\right) & -r_{i}\left(\lambda+p_{i}\right) \\
r_{i}\left(\lambda+p_{i-1}\right) & -\frac{1}{2}\left(\lambda^{2}-p_{i-1}^{2}-R_{i-1}+R_{i}\right)
\end{array}\right)\left(\begin{array}{c}
\psi_{i} \\
\psi_{i-1}
\end{array}\right)
$$

В частности, из условия (33) следует, что $\operatorname{Tr} A_{2}(\lambda)=0$.

Для оператора (34) уравнение кривой (20) принимает вид

$$
\begin{gathered}
y^{2}=\left(\lambda^{2}-\frac{1}{2} Q\right)^{2}-4 R_{i}\left(\lambda+p_{i}\right)\left(\lambda+p_{i-1}\right), \\
Q \equiv R_{i+1}+R_{i-1}-2 R_{i}+p_{i}^{2}+p_{i-1}^{2}
\end{gathered}
$$

Разлагая производящий дифференциал при $\lambda \rightarrow \infty$, получаем с учетом равенства $Q+4 R_{i}=2 C_{2}$, что

$$
\begin{aligned}
y d \lambda \underset{\lambda \rightarrow \infty}{\sim} & d \lambda\left(\lambda^{2}-\frac{1}{2}\left(Q+4 R_{i}\right)-\frac{2 R_{i}}{\lambda}\left(p_{i}+p_{i-1}\right)-\frac{R_{i}}{\lambda^{2}}\left(2 p_{i} p_{i-1}+Q+2 R_{i}\right)+\cdots\right)= \\
= & d \lambda\left(\lambda^{2}-C_{2}-\frac{2 C_{1}}{\lambda}-\frac{2 R_{i}}{\lambda^{2}}\left(p_{i} p_{i-1}-R_{i}+C_{2}\right)-\right. \\
& \left.-\frac{2 C_{1} C_{2}}{\lambda^{3}}-\frac{2}{\lambda^{4}}\left(C_{1}^{2}+C_{2} H\right)+\cdots\right) .
\end{aligned}
$$

Принимая во внимание соотношение $2 C_{2}=R_{i+1}+R_{i-1}+2 R_{i}+p_{i}^{2}+p_{i-1}^{2}$ и коэффициент при $d \lambda / \lambda$, приходим к следующей форме казимиров:

$$
C_{1}=R_{i}\left(p_{i}+p_{i-1}\right), \quad C_{2}=R_{i+1}+R_{i}+p_{i}^{2}=R_{i-1}+R_{i}+p_{i-1}^{2},
$$


которые вместе с равенствами (33) редуцируют фазовое пространство до двумерного. Удобно в качестве координат в этом пространстве выбрать $R_{i}$ (для некоторого фиксированного узла $i$ ) и разность соответствующих импульсов $p_{-}=p_{i}-p_{i-1}$.

Гамильтонианом $H$ является с точностью до численного множителя коэффициент при члене $d \lambda / \lambda^{2}$ разложения (36),

$$
H=R_{i}\left(p_{i} p_{i-1}-R_{i}+C_{2}\right)=\frac{C_{1}^{2}}{4} \frac{1}{R_{i}}-\frac{1}{4} R_{i} p_{-}^{2}-R_{i}+C_{2} R_{i} .
$$

Принимая во внимание равенства (37), уравнение кривой (35) можно переписать в виде

$$
y^{2}=\left(\lambda^{2}-C_{2}\right)^{2}-4\left(C_{1} \lambda+H\right),
$$

так что оно в точности совпадает с кривой (3) матричной модели с потенциалом $W=\lambda^{3} / 3-C_{2} \lambda$ и функцией $f_{1}=C_{1} \lambda+H$.

Явное вычисление симплектической формы для случая $n=2$ на основании формул (23) и (19) с учетом (37) приводит к равенствам

$$
\begin{aligned}
\Omega_{2}= & \frac{1}{2} d\left(R_{i-1}+R_{i}+p_{i-1}^{2}\right) \wedge d \xi_{i-1}^{-}- \\
& \quad-\frac{1}{2} d\left(R_{i+1}+R_{i}+p_{i}^{2}\right) \wedge d \xi_{i}^{+}+d\left(p_{i}-p_{i-1}\right) \wedge d R_{i}= \\
= & d\left(p_{i}-p_{i-1}\right) \wedge d R_{i} .
\end{aligned}
$$

Наконец, чтобы согласовать друг с другом гамильтониан (38), кривую (39) и симплектическую форму (40), следует переписать уравнение (39) в форме, используемой в теории Виттена-Зайберга (см., например, [6] и [12]), т.е. в виде

$$
w+\frac{C_{1} \lambda+H}{w}=\lambda^{2}-C_{2}, \quad w-\frac{C_{1} \lambda+H}{w}=y .
$$

Это означает, в частности, что $2 w=y+\lambda^{2}-C_{2}$ и

$$
\frac{1}{2} d y \wedge d \lambda=d w \wedge d \lambda \text {. }
$$

Первое из уравнений (41) может теперь быть переписано как

$$
H=w \lambda^{2}-C_{1} \lambda-C_{2} w-w^{2}=w\left(\lambda-\frac{C_{1}}{2 w}\right)^{2}-\frac{C_{1}^{2}}{4 w}-C_{2} w-w^{2},
$$

что в точности совпадает с формулой (38), если произвести отождествления

$$
w \leftrightarrow-R_{i}, \quad \lambda-\frac{C_{1}}{2 w} \leftrightarrow \pm p_{-} .
$$

4.3. Случай $n=3$. Для случая $n=3$ при $c_{k} \propto \delta_{k 3}$ явное выражение для оператора $A_{3}=\mathcal{R} \circ L^{3} / 2$ принимает вид

$$
\left(A_{3} \psi\right)_{i} \propto r_{i+3} r_{i+2} r_{i+1} \psi_{i+3}+r_{i+2} r_{i+1}\left(p_{i+2}+p_{i+1}+p_{i}\right) \psi_{i+2}+
$$




$$
\begin{aligned}
& +r_{i+1}\left(p_{i+1}^{2}+p_{i+1} p_{i}+p_{i}^{2}+r_{i+2}^{2}+R_{i+1}+R_{i}\right) \psi_{i+1}- \\
& -r_{i}\left(p_{i}^{2}+p_{i} p_{i-1}+p_{i-1}^{2}+R_{i+1}+R_{i}+R_{i-1}\right) \psi_{i-1}- \\
& -r_{i-1} r_{i-2}\left(p_{i}+p_{i-1}+p_{i-2}\right) \psi_{i-2}-r_{i} r_{i-1} r_{i-2} \psi_{i-3},
\end{aligned}
$$

в то время как стационарность градиента следовой части

$$
\frac{1}{3} \operatorname{Tr} L^{3}=\frac{1}{3} \sum_{i} p_{i}^{3}+\sum_{i} p_{i}\left(R_{i}+R_{i+1}\right)
$$

приводит к условиям (33) предыдущего примера. Условие коммутативности (16) теперь может быть записано как

$$
\begin{gathered}
p_{i}^{3}+\left(p_{i+1}+2 p_{i}\right) R_{i+1}+p_{i} R_{i} p_{i-1}^{3}+\left(p_{i-2}+2 p_{i-1}\right) R_{i-1}+p_{i-1} R_{i}= \\
=R_{i+1}\left(p_{i+1}^{2}+p_{i+1} p_{i}+p_{i}^{2}+R_{i+2}+R_{i+1}+R_{i}\right) \times \\
\times R_{i}\left(p_{i}^{2}+p_{i} p_{i-1}+p_{i-1}^{2}+R_{i+1}+R_{i}+R_{i-1}\right) .
\end{gathered}
$$

В базисе собственных функций оператора Лакса оператор $A_{3}$ в (45) представляется матрицей с элементами

$$
\begin{aligned}
\left(A_{3}(\lambda)_{11}\right. & =\frac{1}{2}\left(\lambda^{3}+2 r_{i}^{2} \lambda-p_{i}^{3}-\left(p_{i+1}+2 p_{i}\right) r_{i+1}^{2}+p_{i-1} r_{i}^{2}\right), \\
\left(A_{3}(\lambda)\right)_{12} & =-r_{i}\left(\lambda^{2}+p_{i} \lambda+p_{i}^{2}+r_{i+1}^{2}+r_{i}^{2}\right), \\
\left(A_{3}(\lambda)\right)_{21} & =r_{i}\left(\lambda^{2}+p_{i-1} \lambda+p_{i-1}^{2}+r_{i}^{2}+r_{i-1}^{2}\right), \\
\left(A_{3}(\lambda)\right)_{22} & =-\frac{1}{2}\left(\lambda^{3}+2 r_{i}^{2} \lambda-p_{i-1}^{3}-\left(p_{i-2}+2 p_{i-1}\right) r_{i-1}^{2}+p_{i} r_{i}^{2}\right),
\end{aligned}
$$

а уравнение кривой (20) для оператора (48) принимает вид

$$
\begin{aligned}
y^{2} \lambda^{6}=( & \left.p_{i}^{3}+p_{i-1}^{3}+\left(p_{i-2}+2 p_{i-1}\right) R_{i-1}+3\left(p_{i}+p_{i-1}\right) R_{i}+\left(p_{i+1}+2 p_{i}\right) R_{i+1}\right) \lambda^{3}- \\
& -4 R_{i}\left(R_{i+1}+R_{i}+R_{i-1}+p_{i}^{2}+p_{i} p_{i-1}+p_{i-1}^{2}\right) \lambda^{2}- \\
& -2 R_{i}\left(\left(p_{i+1}+2 p_{i}+2 p_{i-1}\right) R_{i+1}+\left(p_{i}+p_{i-1}\right) R_{i}+\right. \\
& \left.+\left(p_{i-2}+2 p_{i-1}+2 p_{i}\right) R_{i-1}+p_{i}^{3}+2 p_{i}^{2} p_{i-1}+2 p_{i} p_{i-1}^{2}+p_{i-1}^{3}\right) \lambda+ \\
& +\frac{1}{4}\left(\left(p_{i+1}+2 p_{i}\right) R_{i+1}-\left(p_{i}+p_{i-1}\right) R_{i}+\left(p_{i-2}+2 p_{i-1}\right) R_{i-1}+p_{i}^{3}+p_{i-1}^{3}\right)^{2}- \\
& -4 R_{i}\left(p_{i}^{2}+R_{i+1}+R_{i}\right)\left(p_{i-1}^{2}+R_{i}+R_{i-1}\right) .
\end{aligned}
$$

Разлагая производящий дифференциал, получим

$$
\begin{aligned}
y d \lambda \underset{\lambda \rightarrow \infty}{\sim} & d \lambda\left(\lambda^{3}-\frac{1}{2}\left(\left(p_{i+1}+2 p_{i}\right) R_{i+1}+3\left(p_{i}+p_{i-1}\right) R_{i}+\left(p_{i-2}+2 p_{i-1}\right) R_{i-1}+\right.\right. \\
& \left.+p_{i}^{3}+p_{i-1}^{3}\right)-\frac{2 R_{i}}{\lambda}\left(R_{i+1}+R_{i}+R_{i-1}+p_{i}^{2}+p_{i} p_{i-1}+p_{i-1}^{2}\right)- \\
& -\frac{R_{i}}{\lambda^{2}}\left(\left(p_{i+1}+2 p_{i}+2 p_{i-1}\right) R_{i+1}++\left(p_{i}+p_{i-1}\right) R_{i}+\right. \\
& \left.+\left(p_{i-2}+2 p_{i-1}+2 p_{i}\right) R_{i-1}+p_{i}^{3}+2 p_{i}^{2} p_{i-1}+2 p_{i} p_{i-1}^{2}+p_{i-1}^{3}\right)-
\end{aligned}
$$




$$
\begin{aligned}
& -\frac{R_{i}}{\lambda^{3}}\left(\left(p_{i+1} p_{i}+p_{i+1} p_{i-1}+2 p_{i}^{2}+2 p_{i} p_{i-1}+\right.\right. \\
& \left.+2 p_{i-1}^{2}\right) R_{i+1}+\left(3 p_{i}^{2}+3 p_{i-1}^{2}+2 p_{i} p_{i-1}\right) R_{i}+ \\
& +\left(p_{i-1} p_{i-2}+p_{i} p_{i-2}+2 p_{i} p_{i-1}+2 p_{i-1}^{2}+2 p_{i}^{2}\right) R_{i-1}+ \\
& +2\left(R_{i+1} R_{i}+R_{i+1} R_{i-1}+R_{i}^{2}+R_{i} R_{i-1}\right)+ \\
& \left.\left.+p_{i}^{4}+p_{i}^{3} p_{i-1}+2 p_{i}^{2} p_{i-1}^{2}+p_{i} p_{i-1}^{3}+p_{i-1}^{4}\right)+\cdots\right) .
\end{aligned}
$$

Полагая, что коэффициенты при сингулярных членах разложения $y d \lambda$ являются числами, не зависящими от динамических параметров (казимирами), и используя (48), имеем

$$
\begin{gathered}
C_{1}=R_{i}\left(p_{i}^{2}+p_{i} p_{i-1}+p_{i-1}^{2}+R_{i+1}+R_{i}+R_{i-1}\right), \\
C_{3}=p_{i}^{3}+\left(p_{i+1}+2 p_{i}\right) R_{i+1}+\left(p_{i-1}+2 p_{i}\right) R_{i} p_{i-1}^{3}+ \\
+\left(p_{i-2}+2 p_{i-1}\right) R_{i-1}+\left(2 p_{i-1}+p_{i}\right) R_{i} .
\end{gathered}
$$

Вместе с условиями (47) соотношения (51) налагают четыре связи на восьмимерное пространство переменных $\left(R_{j}\right.$ и $p_{j}$ при $j=i-1, i, i+1$, а также $R_{i-2}$ и $\left.p_{i+2}\right)$, так что в данном примере фазовое пространство является четырехмерным.

Таким образом, разложение (50) с учетом равенств (51) может быть переписано как

$$
\begin{aligned}
y d \lambda \underset{\lambda \rightarrow \infty}{\sim} & d \lambda\left(\lambda^{3}-C_{3}-\frac{2 R_{i}}{\lambda}\left(R_{i+1}+R_{i}+R_{i-1}+p_{i}^{2}+p_{i} p_{i-1}+p_{i-1}^{2}\right)-\right. \\
& \left.-\frac{2 R_{i}}{\lambda^{2}}\left(p_{i-1} R_{i+1}-\left(p_{i}+p_{i-1}\right) R_{i}+p_{i} R_{i-1}+p_{i}^{2} p_{i-1}+p_{i} p_{i-1}^{2}+C_{3}\right)+\cdots\right)= \\
= & d \lambda\left(\lambda^{3}-C_{3}-\frac{2 C_{1}}{\lambda}-\frac{2 H_{1}}{\lambda^{2}}-\frac{2 H_{2}}{\lambda^{3}}+\cdots\right),
\end{aligned}
$$

так что первые два независимых гамильтониана (пропорциональные коэффициентам при $d \lambda / \lambda^{2}$ и $d \lambda / \lambda^{3}$ ) могут быть с помощью (51) представлены в виде

$$
\begin{aligned}
H_{1}= & C_{3} R_{i}+\frac{C_{1}}{2}\left(p_{i}+p_{i-1}\right)- \\
& \quad-\frac{1}{2} R_{i}\left(p_{i}^{3}+p_{i-1}^{3}+3 R_{i}\left(p_{i}+p_{i-1}\right)+\left(p_{i}-p_{i-1}\right)\left(R_{i+1}-R_{i-1}\right)\right), \\
H_{2}= & \frac{C_{1}^{2}}{4 R_{i}}+\frac{C_{1}}{2}\left(R_{i}-p_{i} p_{i-1}\right)+C_{3} R_{i}\left(p_{i}+p_{i-1}\right)+\frac{1}{4} R_{i}^{3}- \\
& \quad-\frac{1}{4} R_{i}\left(R_{i+1}-R_{i-1}\right)^{2}-\frac{1}{2} R_{i}\left(R_{i+1}-R_{i-1}\right)\left(p_{i}^{2}-p_{i-1}^{2}\right)- \\
& -\frac{1}{4} R_{i}\left(p_{i}^{4}+3 p_{i}^{2} p_{i-1}^{2}+p_{i-1}^{4}\right)-R_{i}^{2}\left(p_{i}^{2}+\frac{5}{3} p_{i} p_{i-1}+p_{i-1}^{2}\right) .
\end{aligned}
$$

Уравнение кривой (49) теперь превращается в

$$
y^{2}=\left(\lambda^{3}-C_{3}\right)^{2}-4\left(C_{1} \lambda^{2}+H_{1} \lambda+H_{2}\right),
$$


вновь совпадая с уравнением кривой для матричной модели (3) с потенциалом $W=$ $\lambda^{4} / 4-C_{3} \lambda$ и функцией $f_{2}=C_{1} \lambda^{2}+H_{1} \lambda+H_{2}$.

Явное вычисление симплектической формы $\Omega_{3}$ приводит к выражению

$$
\begin{aligned}
\Omega_{3}=\frac{1}{2} & d \xi_{i-1}^{-} \wedge d\left(p_{i-1}^{3}+\left(p_{i-2}+2 p_{i-1}\right) R_{i-1}+\left(p_{i}+2 p_{i-1}\right) R_{i}\right)+ \\
& +\frac{1}{2} d\left(p_{i}^{3}+\left(p_{i+1}+2 p_{i}\right) R_{i+1}+\left(p_{i-1}+2 p_{i}\right) R_{i}\right) \wedge d \xi_{i}^{+} \\
& +d\left(R_{i+1}-R_{i-1}\right) \wedge d R_{i}+2 R_{i} d p_{i} \wedge d p_{i-1}+ \\
& +d\left(p_{i}^{2}-p_{i-1}^{2}\right) \wedge d R_{i}+\left(p_{i-1} d p_{i}-p_{i} d p_{i-1}\right) \wedge d R_{i} .
\end{aligned}
$$

Используя (51) и вводя $R_{-} \equiv R_{i+1}-R_{i-1}$, это выражение можно слегка упростить до

$$
\begin{aligned}
\Omega_{3}= & d R_{-} \wedge d R_{i}+2 R_{i} d p_{i} \wedge d p_{i-1}+ \\
& +d\left(p_{i}^{2}-p_{i-1}^{2}\right) \wedge d R_{i}+\left(p_{i-1} d p_{i}-p_{i} d p_{i-1}\right) \wedge d R_{i}
\end{aligned}
$$

(естественно, $\left.d\left(\Omega_{3}\right)=0\right)$ или

$$
\Omega_{3}=\left(d R_{-} d R_{i} d p_{i} d p_{i-1}\right) \cdot \widehat{\Omega}_{3} \cdot\left(\begin{array}{c}
d R_{-} \\
d R_{i} \\
d p_{i} \\
d p_{i-1}
\end{array}\right)
$$

где подразумевается внешнее произведение, а матрица $\widehat{\Omega}_{3}$ имеет вид

$$
\widehat{\Omega}_{3}=\left(\begin{array}{cccc}
0 & \frac{1}{2} & 0 & 0 \\
-\frac{1}{2} & 0 & -\left(p_{i}+\frac{p_{i-1}}{2}\right) & p_{i-1}+\frac{p_{i}}{2} \\
0 & p_{i}+\frac{p_{i-1}}{2} & 0 & R_{i} \\
0 & -\left(p_{i-1}+\frac{p_{i}}{2}\right) & -R_{i} & 0
\end{array}\right) .
$$

Нетривиальной является проверка того, что гамильтонианы (53) и (54) действительно коммутируют:

$$
\left\{H_{1}, H_{2}\right\}_{\Omega_{3}}=0
$$

относительно скобки Пуассона, отвечающей симплектической структуре (57), т.е. определяемой обратной к (59) матрицей

$$
\left(\begin{array}{cccc}
0 & -2 & \frac{2 p_{i-1}+p_{i}}{R_{i}} & \frac{2 p_{i}+p_{i-1}}{R_{i}} \\
2 & 0 & 0 & 0 \\
-\frac{2 p_{i-1}+p_{i}}{R_{i}} & 0 & 0 & -\frac{1}{R_{i}} \\
-\frac{2 p_{i}+p_{i-1}}{R_{i}} & 0 & \frac{1}{R_{i}} & 0
\end{array}\right)
$$


приводящей к “элементарным” скобкам

$$
\begin{aligned}
\left\{R_{i}, R_{-}\right\}_{\Omega_{3}} & =1, \\
\left\{p_{i}, p_{i-1}\right\}_{\Omega_{3}} & =-\frac{1}{2 R_{i}}, \\
\left\{R_{-}, p_{i}\right\}_{\Omega_{3}} & =\frac{1}{R_{i}}\left(p_{i-1}+\frac{p_{i}}{2}\right), \\
\left\{R_{-}, p_{i-1}\right\}_{\Omega_{3}} & =\frac{1}{R_{i}}\left(p_{i}+\frac{p_{i-1}}{2}\right) .
\end{aligned}
$$

Симплектическую структуру (57) можно привести к канонической форме, вводя новые переменные так, что $p_{i}=p \operatorname{ch} \theta$ и $p_{i-1}=p \operatorname{sh} \theta$, тогда

$$
\begin{aligned}
\Omega_{3} & =d R_{-} \wedge d R_{i}+R_{i} d\left(p^{2}\right) \wedge d \theta+p^{2} d R_{i} \wedge d \theta+d\left(p^{2}\right) \wedge d R_{i}= \\
& =d\left(R_{-}+p^{2}\right) \wedge d R_{i}+d\left(R_{i} p^{2}\right) \wedge d \theta,
\end{aligned}
$$

и мы приходим к выводу, что переменными Дарбу являются $\theta, R \equiv R_{i}$ вместе с

$$
\rho=R_{i} p^{2}, \quad \Delta=R_{-}+p^{2} .
$$

Для этих переменных скобки Пуассона имеют вид

$$
\{\Delta, R\}_{\Omega_{3}}=1, \quad\{\rho, \theta\}_{\Omega_{3}}=1
$$

а все остальные равны нулю. Гамильтонианы (53), (54) в этих переменных записываются как

$$
\begin{aligned}
H_{1}= & C_{3} R+\frac{C_{1}}{2} \rho^{1 / 2} R^{-1 / 2} e^{\theta}-\frac{1}{2} \Delta \rho^{1 / 2} R^{1 / 2} e^{-\theta}+ \\
& +\frac{1}{8}\left(e^{-\theta}-e^{3 \theta}\right) \rho^{3 / 2} R^{-1 / 2}-\frac{3}{2} \rho^{1 / 2} R^{3 / 2} e^{\theta} \\
H_{2}= & \frac{C_{1}^{2}}{4 R}+\frac{C_{1}}{2} R+\frac{C_{1}}{8} \frac{\rho}{R}\left(e^{-2 \theta}-e^{2 \theta}\right)+C_{3} \rho^{1 / 2} R^{1 / 2} e^{\theta}+ \\
& +\frac{1}{4} R^{3}-\frac{1}{4} \Delta^{2} R-\frac{1}{32} \frac{\rho^{2}}{R}-\frac{9}{8} \rho R e^{2 \theta}+\frac{1}{8} \rho R e^{-2 \theta}+\frac{1}{64} \frac{\rho^{2}}{R}\left(e^{4 \theta}+e^{-4 \theta}\right),
\end{aligned}
$$

т.е. являются функциями, вообще говоря, от дробных степеней динамических переменных. Зависимость от дробных степеней, однако, пропадает для $H_{2}$ при нулевом казимире $C_{3}=0$ и для квадрата гамильтониана $H_{1}^{2}$ при $C_{1}=C_{3}=0$.

\section{5. ОБСУЖДЕНИЕ}

Обсудим некоторые проблемы, связанные с данной задачей, а также перечислим оставшиеся открытыми вопросы.

Геометрия стационарной и периодической задач. Определяя стационарную задачу, мы исходили из бесконечной цепочки Тоды. Вместо этого, оставляя все рассуждения практически без изменений, в качестве отправной точки можно взять 
$N$-периодическую задачу для цепочки Тоды $\left(q_{i+N}=q_{i}, \quad p_{i+N}=p_{i}, \quad \psi_{i+N}=w \psi_{i}\right.$ и т.п.) при достаточно большом $N$.

Эту процедуру, однако, можно проинтерпретировать полностью в терминах алгебро-геометрических интегрируемых систем. Исходя из кривой $N$-периодической цепочки

$$
w+\frac{1}{w}=P_{N}(\lambda)
$$

оснащенной производящим дифференциалом $d S=\lambda d w / w$, можно рассмотреть редукцию цепочки в $2 n$-мерное подпространство в пространстве модулей при $n<N$. Естественный способ осуществить такую редукцию - потребовать существования на кривой (67) однозначной мероморфной функции с не более чем двумя полюсами (в точках, где $\lambda=\infty$ ) порядка $n$. Существование такой функции на кривой (67) в точности эквивалентно стационарности потока $n$-ого порядка в периодической цепочке Тоды, ассоциированного с линейной комбинацией мероморфных дифференциалов $d \Omega_{A}=\sum_{k=1}^{n} c_{k} d \Omega_{k}$. Поскольку в ситуации общего положения на кривой $(67)$ не существует естественной функции с такими свойствами, это условие эффективно уменьшает (гладкий) род римановой поверхности от $N-1$ до $n-1$, и в результате мы получаем кривую (3) стационарной задачи. Подобная редукция уже обсуждалась в литературе в контексте связей между геометриями Дийкграафа-Вафы и Виттена-Зайберга, см. [3] и, например, [13]. Заметим также, что соответствующие производящие дифференциалы или симплектические структуры остаются при данной процедуре "развязанными", и это отвечает различию в симплектических структурах (21), (40) и (57) стационарной задачи и бесконечной цепочки, которое уже обсуждалось выше.

О квантовой задаче. Глядя на кривую (39) и симплектическую форму (42), можно сразу написать "наивное" уравнение Шредингера

$$
\hbar^{2} \frac{\partial^{2}}{\partial \lambda^{2}} \Upsilon=\left(W^{\prime}(\lambda)^{2}-\hbar W^{\prime \prime}(\lambda)\right) \Upsilon
$$

с решением

$$
\Upsilon=e^{-W(\lambda) / \hbar}
$$

поскольку

$$
\hbar^{2} \frac{\partial^{2}}{\partial \lambda^{2}}-W^{\prime}(\lambda)^{2}+\hbar W^{\prime \prime}(\lambda)=\left(\hbar \frac{\partial}{\partial \lambda}-W^{\prime}(\lambda)\right)\left(\hbar \frac{\partial}{\partial \lambda}+W^{\prime}(\lambda)\right) .
$$

Сравнение формул (68) и (39) для потенциала $W^{\prime}(\lambda)=\lambda^{2}-C_{2}$ дает $H=0$ и $C_{1}=\hbar / 2$, а сравнение с уравнением (55) приводит к $H_{2}=H_{1}=0$ и $C_{1}=3 \hbar / 4$. Формулы (41) и (42) позволяют думать, что квантовать систему лучше в переменных $(y, w)$. Для периодической цепочки Тоды (67) подобный путь приводит к уравнению Бакстера (разностному уравнению второго порядка), но для стационарной задачи из уравнения (41) в силу (42) в уравнении Бакстера появляется интегральный оператор. Главным препятствием на этом пути является неясность с выбором “правильных" переменных для квантования стационарной задачи, так как в наивных 
переменных Дарбу уже в случае $n=3$ гамильтонианы являются, вообще говоря, неаналитическими функциями.

Тем не менее можно попытаться решать квантовую задачу

$$
\widehat{\mathcal{H}}_{i} \mathcal{Z}=H_{i} \mathcal{Z}, \quad i=1, \ldots, n-1,
$$

для матричномодельной $\mathcal{Z}$-функции, которая в отличие от изначальной статсуммы матричной модели $Z$, определенной интегральным представлением (1), содержит явную информацию о (квантовом аналоге) геометрии (3), (4), т.е. буквально следует искать решение (71) для функции

$$
\mathcal{Z}(c, H)=\exp \left(\frac{1}{\hbar^{2}} F_{0}(c, H)+\cdots\right) .
$$

Такая постановка задачи согласована с тем фактом, что вид гамильтонианов (66) упрощается для нулевых (классических) казимиров, т.е. при условии, что их значения пропорциональны $\hbar$ (являются квантовыми поправками). При этом классические формулы (66) для гамильтонианов $H_{i}$ (или некоторых полиномиальных функций от них) превращаются в дифференциальные или разностные операторы $\widehat{\mathcal{H}}_{i}$ посредством замены $\Delta \rightarrow \partial / \partial R$ и $\theta \rightarrow \partial / \partial \rho$, так что $e^{ \pm \theta}$ являются операторами сдвига по переменной $\rho$. Определенная таким образом $\mathcal{Z}$-функция $(72)$ является буквальным матричномодельным аналогом некрасовского обобщения [14] препотенциала Виттена-Зайберга.

Благодарности. Автор признателен Г. Брадену, А. Веселову, Р. Донаги, Х. Канно, А. Миронову, Н. Некрасову, М. Ольшанецкому, В. Рубцову, С. Харчёву, Б. Хесину и особенно И. Кричеверу за полезные обсуждения. Работа была выполнена при частичной поддержке РФФИ (грант № 04-01-00642), Программы поддержки научных школ (грант № 1578.2003.2), Федеральной программы Министерства промышленности, науки и технологий РФ (грант № 40.052.1.1.1112) и Фонда поддержки Российской науки.

\section{Список литературы}

[1] A. A. Migdal. Phys. Rep. 1983. V. 102. P. 199.

[2] F. David. Phys. Lett. B. 1993. V. 302. P. 403-410; hep-th/9212106; G. Bonnet, F. David, B. Eynard. J. Phys. A. 2000. V. 33. P. 6739-6768; cond-mat/0003324.

[3] F. Cachazo, K. Intriligator, C. Vafa. Nucl. Phys. B. 2001. V. 603. P. 3-41; hepth/0103067; F. Cachazo, C. Vafa. $N=1$ and $N=2$ geometry from fluxes. hep-th/0206017.

[4] R. Dijkgraaf, C. Vafa. Matrix models, topological strings, and supersymmetric gauge theories. hep-th/0206255; On geometry and matrix models. hep-th/0207106; A perturbative window into non-perturbative physics. hep-th/0208048.

[5] I. Krichever. Commun. Pure Appl. Math. 1992. V. 47. P. 437; hep-th/9205110.

[6] A. Marshakov. Seiberg-Witten Theory and Integrable Systems. Singapore: World Scientific, 1999.

[7] Integrability: The Seiberg-Witten and Whitham Equations. Eds. H. W. Braden, I. M. Krichever. Amsterdam: Gordon and Breach, 2000. 
[8] A. Gerasimov, A. Marshakov, A. Mironov, A. Morozov, A. Orlov. Nucl. Phys. B. 1991. V. 357. P. 565; S. Kharchev, A. Marshakov, A. Mironov, A. Orlov, A. Zabrodin. Nucl. Phys. B. 1991. V. 366. P. 569.

[9] A. Gorsky, I. Krichever, A. Marshakov, A. Mironov, A. Morozov. Phys. Lett. B. 1995. V. 355. P. 466-477; hep-th/9505035.

[10] О. И. Богоявленский, С. П. Новиков. Функц. анализ и его прилож. 1976. Т. 10. № 1. С. 9; В.Е. Захаров, С.В. Манаков, С.П. Новиков, П. П. Питаевский. Теория солитонов: метод обратной задачи. М.: Наука, 1980; Б. А. Дубровин, И. М. Кричевер, С. П. Новиков. Интегрируемые системы. В сб.: Итоги науки и техники. Сер. Современные проблемы математики. Фундаментальные направления. Т. 4. Ред. Р. В. Гамкрелидзе, В. И. Арнольд, С. П. Новиков. М.: ВИНИТИ, 1985. C. 179 .

[11] I. M. Krichever, D. H. Phong. J. Diff. Geom. 1997. V. 45. P. 349; hep-th/9604199; Symplectic forms in the theory of solitons. hep-th/9708170; A. Marshakov. From nonperturbative SUSY gauge theories to integrable systems. In: Proc. 10th Int. Conf. "Problems of Quantum Field Theory" (Dubna, 1996); hep-th/9607159.

[12] A. Marshakov. Mod. Phys. Lett. A. 1996. V. 11. P. 1169; hep-th/9602005; A. Gorsky, A. Marshakov, A. Mironov, A. Morozov. Phys. Lett. B. 1996. V. 380. P. 75; hep-th/9603140.

[13] T. J. Hollowood. JHEP. 2003. V. 0310. P. 051; hep-th/0305023; R. Boels, J. de Boer, R. Duivenvoorden, J. Wijnhout. JHEP. 2004. V. 0403. P. 010; hep-th/0305189; H. Itoyama, H. Kanno. Nucl. Phys. B. 2004. V. 686. P. 155; hep-th $/ 0312306$.

[14] N. A. Nekrasov. Adv. Theor. Math. Phys. 2004. V. 7. P. 831; hep-th/0206161; Z theory. hep-th/0412021; A. S. Losev, A. Marshakov, N. A. Nekrasov. Small instantons, little strings and free fermions. hep-th/0302191; N. Nekrasov, A. Okounkov. Seiberg-Witten theory and random partitions. hep-th/0306238; A. Braverman. Instanton counting via affine Lie algebras. I: Equivariant J-functions of (affine) flag manifolds and Whittaker vectors. math.AG/0401409; A. Braverman, P. Etingof. Instanton counting via affine Lie algebras II: from Whittaker vectors to the Seiberg-Witten prepotential. math.AG/0409441. 